\title{
Ecología trófica, dimorfismo sexual y parámetros reproductivos en el lagarto endémico Andino Liolaemus vallecurensis, Argentina
}

\author{
Gabriel N. Castillo',2, Héctor J. Villavicencio', Juan C. Acosta' \& José Marinero'
}

\begin{abstract}
1. Departamento de Biología, Facultad de Ciencias Exactas, Físicas y Naturales, Universidad Nacional de San Juan. Av. Ignacio de la Roza 590, 5402 , San Juan, Argentina. (nataliocastillo@gmail.com, hjvillavicencio@gmail.com, jcacostasanjuan@gmail.com)

2. Becario de CONICET (Consejo Nacional de Investigaciones Científicas y Técnicas). Departamento de Biología, Universidad Nacional de San Juan. Av. Ignacio de la Roza 590, 5402, San Juan, Argentina.
\end{abstract}

Received 30 November 2016

Accepted 23 November 2017

$10.1590 / 1678-4766 \mathrm{e} 2017046$

\begin{abstract}
Trophic ecology, sexual dimorphism and reproductive parameters in the endemic Andean lizard Liolaemus vallecurensis, Argentina. The feeding habits, sexual dimorphism and reproductive parameters of Liolaemus vallecurensis (Pereyra, 1992) were assessed in an Andean sector of Argentina. We determined the total volume, number, and occurrence frequency of each prey item and calculated the relative importance indexes. Trophic indices (Levins) and diversity indices were applied as well. Also studied were morphological variations in 14 measurements, comparing adult males and females. In concerning arthropods, L. vallecurensis feeds mostly on items from the order Hymenoptera (not Formicidae), with no differences being found between sexes or ages. Males show a tendency towards an active search strategy and a specialized diet. Females and juveniles hunt by stalking, and have a more opportunistic diet. Males consume large amounts of plant matter considered as herbivores but not strictly, females are omnivores and juveniles between insectivores and omnivores. Dimorphism was found between sexes for 10 morphological variables. Head length and food search mode were found to be associated. A viviparous condition is confirmed by a clutch size of three young, with parturition occurring between late December and early January. These contributions to L. vallecurensis represent the first and only biological data on this species in these harsh environments.
\end{abstract}

KEYWORDS. Diet, Widely Forraging, specialists, generalists.

RESUMEN. Se evaluaron los hábitos alimenticios, dimorfismo sexual y parámetros reproductivos en Liolaemus vallecurensis (Pereyra, 1992) en un sector Andino de Argentina. Para examinar la dieta, se determinó volumen, numerosidad y frecuencia de ocurrencia para cada ítem-presa y se calculó el Índice de Importancia Relativa (IRI). Además se aplicaron índices tróficos (Levins) e índices de diversidad. Se estudiaron las variaciones morfológicas en 14 medidas comparando machos y hembras adultos. Considerando los artrópodos, L. vallecurensis consume principalmente ítem del orden Hymenoptera (no Formicidae) no encontrándose diferencias tróficas entre sexos ni por edades. Los machos presentan tendencia hacia una estrategia de búsqueda activa y una dieta especialista. Las hembras y juveniles cazan al acecho con una dieta de tipo más oportunista. Los machos consumen grandes cantidades de materia vegetal considerándose como herbívoros aunque no estrictamente, hembras omnívoras y juveniles entre insectívoros y omnívoros. Se encontró dimorfismo entre sexos en 10 variables morfológicas. Se encontró asociación entre la longitud de cabeza y el modo de búsqueda de alimento. Se confirma la condición vivípara con un tamaño de camada de tres crías, con una parición entre fines de diciembre y principios de enero. Estos aportes en L. vallecurensis representan los primeros y únicos datos biológicos para esta especie en estos ambientes rigurosos.

PALABRAS-CLAVE. Dieta, forrajeo activo, especialistas, generalistas.

Actualmente se han planteado dos estrategias de obtención de alimento. En la estrategia o modo de búsqueda activa "Widely Forraging" se componen de movimientos exploratorios y de caza a sus presas con un consumo de presas localmente numerosas y pequeñas. En la estrategia pasiva o caza al acecho "sit and wait" se caracteriza por ser especies oportunistas, consumen un menor número de presas solitarias de mayor tamaño (PIANKA, 1982; VitT \& CALDWELL, 2009). Se han generado dos importantes supuestos con respectos a estas estrategias. El primer supuesto menciona que el modo va a estar en función de las diferencias en abundancias y comportamientos de presas. El segundo supuesto indica que gran parte de la variación de estos modos tienen sus orígenes en lo profundo de la filogenia (VITT \& CALDWELL, 2009). En este segundo supuesto los forrajeadores activos pertenecen al clado Autarchoglossa, mientras que los predadores al acecho pertenecen al clado Iguania (VITT \& PIANKA, 2005).

Se ha discutido que la divergencia trófica entre sexos es una posible fuerza selectiva que conduce a la diferenciación morfológica reduciendo la competencia intraespecífica (PIANKA, 1982; FAIRBAIRN, 1997; Cox et al., 2007). Se han propuestos diferentes hipótesis para brindar una explicación a este fenómeno, siendo estás asociadas a una selección sexual, natural (interacciones ecológicas) y selección de fecundidad (Hedrick \& Temeles, 1989; FAIrbairn, 1997; Cox et al., 2007; VinCENT \& Herrel, 2007). El principal inconveniente radica en que estos tres mecanismos conducen al mismo fenómeno y en ocasiones puede resultar problemático (ANDERSON \& VITT, 1990; Pincheira-Donoso, 2012). Un supuesto interesante que se 
desarrolló con respecto al comportamiento en la dieta es que la morfología craneal puede predecir el modo de búsqueda de alimento (MCBraYer \& CORBIN, 2007). Esta hipótesis predice que las especies con una forma de caza al acecho "sit and wait" poseen cabezas cortas y anchas. En cambio las especies con una forma de búsqueda activa presentan cabezas más largas y estrechas (McBrAYER \& CORBIN, 2007).

La distribución de L. vallecurensis (Liolaemidae) se restringe al Valle del Cura, Departamento de Iglesia. La localidad tipo es "La sepultura" a $3815 \mathrm{msnm}$, siendo una especie endémica de la provincia de San Juan, Argentina (PEREYRA, 1992). La única información biológica disponible para esta especie se encuentra asociada a la temperatura corporal y actividad temporal (CASTILLO et al., 2015) y registro de un endoparásito (GOLDBERG et al., 2004). Esta especie se localiza en una zona con una importante actividad industrial minera, representando potenciales riesgos. Todo lo anteriormente mencionado a determinado que se la categorice como una especie vulnerable para la Argentina (ABDALA et al., 2012).

Con el fin de generar información sobre aspectos de la bioecología desconocidos hasta el momento se plantearon las siguientes interrogantes: 1) ¿Cuáles son las principales categorías de ítems-presas consumidas por $L$. vallecurensis? 2) ¿Cuál es el tipo de dieta (insectívoro, omnívoro y herbívoro) y la especialización (especialista/ oportunista)? 3) ¿Qué estrategia de búsqueda de alimento presenta (Forrajeo activo/ caza al acecho)? 4) ¿Que rasgos morfométricos difieren entre sexos? 5) ¿Existe asociación entre la ecología trófica y el tamaño de cabeza? y 6) ¿Cuál es el modo reproductivo y tamaño de camada?

\section{MATERIALES Y MÉTODOS}

Área de estúdio. El área de estudio se localiza en el Valle del Cura en el extremo norte de la Provincia de San Juan, Argentina. Es una región montañosa, de hasta 5000 ms.n.m (PERUCCA \& MarTos, 2009). Los individuos fueron capturados a $3700 \mathrm{~m}$ de altitud ( $29^{\circ} 19^{\prime} 00^{\prime}$ 'S 69 30 '00”W), en la localidad tipo La Sepultura. La zona corresponde a la región Altoandina, donde dominan matorrales arbustivos bajos y medianos (CABRERA, 1994).

Trabajo de campo. Las tareas de campo se realizaron del 1 al 13 de enero del 2007 en forma continuada e ininterrumpida. Se realizaron recorridos en forma sistemática con revisión aleatoria de arbustos y áreas desprovistas de vegetación (Tellería, 1986). La técnica de captura fue mediante el método de lazo. El esfuerzo de muestreo se mantuvo constante a lo largo de los días y durante cada día. Las recorridas fueron realizadas por dos personas separadas cinco metros una de otras, barriendo un área aproximada de cinco metros a cada lado de cada observador. Los individuos fueron sacrificados inmediatamente después de su captura por administración intraperitoneal de tiopental sódico, fijados al $10 \%$ con formaldehído por 24 horas y finalmente preservados en etanol al $70 \%$. Los individuos colectados se encuentran depositados en la Colección Herpetológica de Zoología de Vertebrados, Universidad Nacional de San Juan.

Análisis de laboratorio. Para la ecología trófica debido a que $L$. vallecurensis es considerada una especie vulnerable se trabajó con una muestra mínima. Se diseccionaron 28 individuos (11 machos, 8 hembras, 9 juveniles). Para la categorización de la dieta se estudiaron los tipos y cantidad de presas bajo lupa binocular estereoscópica. Para artrópodos se midieron longitud (L) y ancho máximo del cuerpo (A) de las presas, y se calculó el volumen individual (V) de las mismas de acuerdo a lo propuesto por Dunham (1983). Para la cuantificación vegetal (tallos, hojas, flores, frutos y semillas), se calculó el porcentaje de materia vegetal que ocupa en el estómago en función del que ocupan las presas artrópodos. El contenido del estómago fue volcado en una caja de petri, observado bajo lupa binocular.

Para el dimorfismo sexual se registraron 14 medidas morfológicas en 32 individuos adultos (14 machos y 18 hembras) usando un calibre Vernier $(0,01 \mathrm{~mm})$ : longitud de cabeza (LCA), ancho de cabeza (ACA), alto de cabeza (ALTCAB), ancho de la base de la cola (ABCO), largo de cola (LCO), longitud del húmero ( $\mathrm{LH})$, largo radio - ulna (LRU), longitud de mano (LM), longitud del fémur (LF), longitud tibia-fíbula (LTF), longitud hocico-cloaca (LHC), distancia entre miembros (DEM), longitud de pata (LP) y alto del cuerpo (ALCUE). Utilizando el tamaño de cabeza (largo y ancho) se las asoció a las estrategias de búsqueda de alimento (MCBRAYER \& CORBIN 2007), donde cabezas largas y estrechas se asocian a un modo de búsqueda activa. Cabezas cortas y anchas se asocian a un modo de búsqueda al acecho.

Para la biología reproductiva fueron analizados los órganos reproductivos de 34 ejemplares (11 hembras y 23 machos). En machos se midió el largo y ancho testicular derecho con un calibre tipo vernier, el cual se obtuvo el volumen. Los tamaños de los testículos se utilizaron como evidencia de actividad reproductiva y en hembras la presencia y tamaño de los folículos. Además se consideraron el número de crías para establecer el tamaño de camada. Para clasificar el nivel de desarrollo alcanzado por el embrión se siguió la propuesta de LEYTON et al. (1980) donde clasificaron los estados de desarrollo en un estado inicial (estadio 1 al 19), medio (20 al 33) y avanzado (34 al 35).

Análisis de los datos. Para determinar la importancia de cada categoría alimentaria se calculó el índice de importancia relativa IRI (PINKAS et al., 1971). Si el porcentaje de IRI de la categoría queda entre el $100 \%$ y el $75 \%$ se la considerará fundamental, entre el $75 \%$ y el $50 \%$ secundaria, entre el $50 \%$ y el $25 \%$ accesoria y si se encuentra en menos del 25\% accidental (Aun \& MARTORI, 1998). Se analizó el material vegetal de los estómagos (tallos, hojas, flores, frutos y semillas) siguiendo a EsPINOZA et al. (2004) modificado de acuerdo a AsTUDILLO et al. (2015) y CóRDOBA et al. (2015) (0-10\% insectívoro, $11-50 \%$ omnívoro; $51-100 \%$ herbívoro). A cada uno de esos valores porcentuales de materia vegetal 
se les fue transformado en categorías ordinales de acuerdo CASTRO et al. (2013) y estas categorías fueron analizadas con la prueba no paramétrica de Kruskal-Wallis.

Para analizar la composición de ítems tróficos entre machos, hembras y juveniles se usó el índice de Jaccard (Moreno, 2001). Como índice de dominancia se usó el índice de Simpson (D) (Moreno, 2001). Como cálculo de diversidad de ítems tróficos se aplicaron los índices de Shannon-Wienner $\left(\mathrm{H}^{\prime}\right)$ y de Margalef $\left(\mathrm{D}_{\mathrm{mg}}\right)$ (Moreno, 2001). Para determinar la amplitud del nicho trófico entre machos, hembras y juveniles se aplicó el índice de Levins. Este índice es máximo cuando las especies son oportunistas, un valor bajo implica especies especialistas (GonZÁlez et al., 2006). Para determinar el modo de búsqueda de alimento se tuvo en cuenta el criterio del consumo de ítems presas. Para el dimorfismo sexual a cada una de las variables se le realizaron estadísticas descriptivas (media, desvío estándar). Para descartar el efecto del tamaño medido como LHC (Largo hocico - cloaca) con cada uno de las variables se probó la dependencia mediante un análisis de regresión lineal simple. Para las variables que no fueran significativas se empleó ANOVA (análisis de la varianza). En el caso de que el tamaño (LHC) influyera en el resto de las variables se empleó ANCOVA (análisis de la covarianza). Para los análisis de ANOVA y ANCOVA se realizaron comparaciones entre machos y hembras adultos. Se realizaron correlaciones de Spearman entre el tamaño de presas (largo, ancho y volumen) con el tamaño de cabeza (largo, ancho, alto) y longitud hocico - cloaca (LHC). El nivel de significancia de los análisis fue de 0,05 y se trabajó con el programa Statistica 10.0 e Infostat y se consultó a ZAR (1996) y SOKAl \& ROHLF (1999). Para el análisis de los parámetros reproductivos, se obtuvieron los valores medios, mínimos y máximos del volumen testicular y del tamaño folicular. Para determinar la relación entre la variable medida para machos (volumen testicular) y hembras (tamaño folicular) con sus respectivos LHC (largo hocico - cloaca), se realizó un análisis de correlación lineal Spearman. Para todos los casos se aseguraron la independencia estadística de los datos.

\section{RESULTADOS}

Ecología trófica. Todos los estómagos aportaron información para el análisis trófico. La riqueza fue 7 ítems para machos, 6 en hembras y 7 en juveniles. La numerosidad, volumen y frecuencias de ocurrencias de presas se observan en la Tab. I. Considerando los artrópodos, machos, hembras y juveniles consumen principalmente el orden Hymenoptera (no Formicidae).

Únicamente en machos se registraron frutos y semillas, estando ausentes en hembras. Machos, hembras y juveniles presentaron materiales vegetales indiferenciados como tallos y hojas. Los machos presentan una tendencia a una herbivoría aunque no estricta $(70,9 \%$ de material vegetal), las hembras omnívoros ( $43,7 \%$ de material vegetal) y juveniles entre insectívoros y omnívoros (16,6\% de material vegetal). Este consumo de material vegetal fue similar en machos y hembras, siendo menor en juveniles (KruskalWallis, $\mathrm{H}=12,9 ; \mathrm{N}=28 ; \mathrm{p}=0,001$ ).

Se encontró diferencia en el análisis de la amplitud del nicho trófico $($ Levins $=\mathrm{Nb})$, presentando valores similares en hembras $(\mathrm{Nb}=4,1)$ y juveniles $(\mathrm{Nb}=4,9)$ y diferentes en machos $(\mathrm{Nb}=1,2)$. Estos resultados indican que los machos presentan una dieta tipo especialista con un consumo de ítems donde la mayor proporción es del $88 \%$ (numerosidad= 212) y corresponden a semillas. Esto es consistente y característico con un modo de búsqueda activo (consumo de presas localmente numerosas y pequeñas). Hembras y juveniles muestran un tipo de dieta oportunista con un consumo menor de presas solitarias de mayor tamaño, siendo esto más característico de un modo de búsqueda al acecho.

El índice de Margalef $\left(\mathrm{D}_{\mathrm{mg}}\right)$ indican valores idénticos entre hembras $(\mathrm{Dmg}=1,4)$ y juveniles $(\mathrm{Dmg}=1,4)$ siendo distintos en machos (Dmg=1,1). El índice de Simpson muestra mayores valores en machos $(D=0,78)$, seguido por hembras $(D=0,2)$ y juveniles $(D=0,17)$. La diversidad [Shannon-Wienner (H')] observada fue mayor en juveniles $\left(H^{\prime}=1,72\right)$, seguido por hembras $\left(H^{\prime}=1,53\right)$ y finalmente machos $\left(H^{\prime}=0,52\right)$. La similitudes en composición de ítemspresas mediante el análisis de Jaccard $=I_{j}$ muestran una baja similitud entre machos, hembras y juveniles [Machos y hembras $(\mathrm{Ij}=0,4)$; machos y juveniles $(\mathrm{Ij}=0,3)$; hembras y juveniles $(\mathrm{Ij}=0,3)]$.

Dimorfismo sexual morfométrico. Las medidas descriptivas se representan en la Tab. II. Las variables se correlacionaron positivamente con el largo hocico-cloaca (LHC), excepto: longitud de la cola (LCO) y longitud del húmero $(\mathrm{LH})$. Del total de las medidas estudiadas no se encontraron diferencias en las siguientes variables: largo radio - ulna (LRU) $\left(\right.$ ANCOVA $\left.\left._{1,29}\right)=1,33, \mathrm{n}=32, \mathrm{p}=0,25\right)$; longitud tibia-fíbula (LTF) (ANCOVA $F_{1,29}=1,174, \mathrm{n}=32, \mathrm{P}=$ $0,28)$; ancho de la base de la cola $(\mathrm{ABCO})\left(\mathrm{ANCOVAF}_{1,29}=\right.$ $1,59, \mathrm{n}=32, \mathrm{P}=0,21$ ); alto del cuerpo (ALCUE) (ANCOVA $\left.\mathrm{F}_{1,29}=2,903, \mathrm{n}=32, \mathrm{P}=0,09\right)$. Todos los valores medios fueron mayores en machos que en hembras.

Se encontraron diferencias siendo todos los valores medios mayores en machos: longitud de cabeza (LCA) (ANCOVA $F_{1,29}=19,59, \mathrm{n}=32, \mathrm{p}=0,0001$ ); ancho de cabeza (ACA) (ANCOVA $F_{1,29}=16,54, n=32, p=0,0003$ ); alto de cabeza (ALTCAB) (ANCOVA $\mathrm{F}_{1,29}=6,05, \mathrm{n}=32, \mathrm{p}=0,02$ ), longitud del fémur (LF) (ANCOVA $\mathrm{F}_{1,29}=5,19, \mathrm{n}=32, \mathrm{p}=$ $0,03)$; longitud de mano (LM) (ANCOVA $\mathrm{F}_{1,29}=6,318, \mathrm{n}=$ $32, \mathrm{p}=0,017)$; longitud de pata (LP) (ANCOVA $\mathrm{F}_{1,29}=4,2$, $\mathrm{n}=32, \mathrm{P}=0,049)$; longitud hocico-cloaca (LHC) (ANOVA $\left.\mathrm{F}_{1,30}=20,98, \mathrm{n}=32, \mathrm{p}=0,0008\right)$; largo de cola (LCO) (ANOVA $\left.\mathrm{F}_{1,30}=10,65, \mathrm{n}=32, \mathrm{P}=0,001\right)$; longitud del húmero $(\mathrm{LH})$ (ANOVA $\mathrm{F}_{1,30}=9,12, \mathrm{n}=32, \mathrm{p}=0,005$ ). No obstante, se encontraron diferencias a favor de las hembras en distancia entre miembros (DEM) (ANCOVA $\mathrm{F}_{1,29}=9,14, \mathrm{n}=32, \mathrm{p}=$ 0,005).

Con respecto a la relación predador - presa, en hembras todas las correlaciones resultaron positivas, pero solamente la correlación entre LCA con el largo, ancho y 


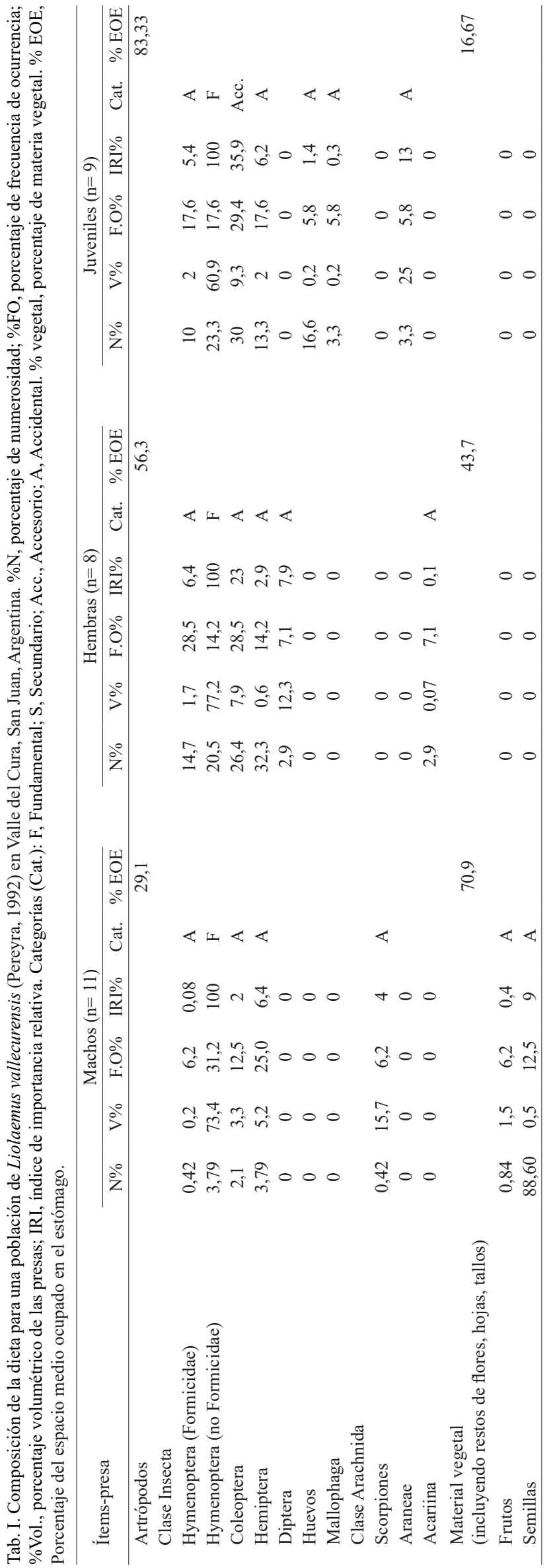

volumen de presa resultaron significativas (Tab. III). Para machos, todas las correlaciones resultaron negativas y no significativas (Tab. III).

Biología reproductiva. Todas las hembras presentaron un tamaño folicular menor a $1 \mathrm{~mm}$ (Media $=0,65 ; \mathrm{Min}=0,60$; Max $=0,90$ ). Los oviductos se encontraban ensanchados, por lo que las hembras se hallaban en estado pos-reproductivo. Los resultados del análisis de correlación no indican relación entre el tamaño del folículo y el LHC $(n=11 ; r=0,47 ; p=$ 0,13 ). En machos se observaron un aumento en los volúmenes testiculares (volumen testicular máximo de $\left.78 \mathrm{~mm}^{3}\right)($ Media $=$ 20,9; $\operatorname{Min}=0,52 ; \operatorname{Max}=78$ ) a partir de un tamaño LHC de $50 \mathrm{~mm}$. El tamaño máximo de LHC fue entre 75 a $80 \mathrm{~mm}$. Se encontró una correlación entre el volumen del testículo

Tab. II. Valores medios y desviación estándar (D.E.) de las medidas de resumen de Liolaemus vallecurensis (Pereyra, 1992) en Valle del Cura, San Juan, Argentina (LCA, longitud de cabeza; ACA, ancho de cabeza; ALTCAB, alto de cabeza; ABCO, ancho de la base de la cola; LCO, Longitud de la cola; LM, longitud de la mano; DEM, distancia entre miembros; LH, longitud del húmero; LRU, longitud radio-ulna; LF, longitud del fémur; LTF, longitud tibia-fíbula; LP, longitud de pata; ALCUE, alto del cuerpo; LHC, longitud hocico-cloaca).

\begin{tabular}{ccccccc}
\hline \multirow{2}{*}{ Variables } & \multicolumn{2}{c}{ Machos $(\mathrm{n}=14)$} & & \multicolumn{2}{c}{ Hembras $(\mathrm{n}=18)$} \\
\cline { 2 - 3 } \cline { 5 - 6 } \cline { 5 - 6 } & Media $(\mathrm{mm})$ & D.E & & Media $(\mathrm{mm})$ & D.E \\
\hline LCA & 15,46 & 1,35 & & 12,05 & 1,37 \\
ACA & 13,35 & 1,54 & & 10,22 & 1,01 \\
ALTCAB & 9,39 & 1,21 & & 7,39 & 0,83 \\
ABCO & 8,61 & 0,84 & & 7,19 & 1,24 \\
LCO & 70,40 & 31,50 & & 55,00 & 15,05 \\
LH & 7,82 & 1,19 & & 6,70 & 0,90 \\
LRU & 5,82 & 1,03 & & 5,42 & 0,92 \\
LM & 10,60 & 0,68 & & 9,03 & 0,80 \\
DEM & 30,02 & 4,13 & & 35,20 & 5,20 \\
LF & 11,43 & 1,12 & & 9,40 & 87,00 \\
LTF & 9,54 & 1,05 & & 8,18 & 0,75 \\
LP & 16,60 & 1,28 & & 14,21 & 1,42 \\
ALCUE & 11,90 & 1,82 & & 8,89 & 1,88 \\
LHC & 66,07 & 6,22 & & 53,80 & 7,90 \\
\hline
\end{tabular}

Tab. III. Análisis de correlación de Spearman (rs) entre el tamaño corporal de Liolaemus vallecurensis (Pereyra, 1992) y variables morfométricas de las presas (LCA, longitud de cabeza; ALCAB, altura de cabeza; ACA, ancho de cabeza; LHC, longitud hocico-cloaca). * indica relaciones estadísticas significativas $(p<0,05)$.

\begin{tabular}{cccccc}
\hline \multirow{2}{*}{ Variables } & \multicolumn{2}{c}{ Machos $(\mathrm{n}=14)$} & & \multicolumn{2}{c}{ Hembras $(\mathrm{n}=15)$} \\
\cline { 2 - 3 } \cline { 6 - 6 } & $\mathrm{r}_{\mathrm{s}}$ & $\mathrm{P}$ & & $\mathrm{r}_{\mathrm{s}}$ & $\mathrm{P}$ \\
\hline LCA x Largo presa & $-0,29$ & 0,30 & & 0,55 & $0,03^{*}$ \\
ACA x Largo presa & $-0,19$ & 0,40 & & 0,26 & 0,34 \\
ALTCAB x Largo presa & $-0,21$ & 0,40 & & 0,45 & 0,09 \\
LHC x Largo presa & $-0,19$ & 0,50 & & 0,20 & 0,46 \\
LCA x Ancho presa & $-0,50$ & 0,06 & & 0,60 & $0,01^{*}$ \\
ACA x Ancho presa & $-0,35$ & 0,20 & & 0,19 & 0,48 \\
ALTCAB Ancho presa & $-0,34$ & 0,20 & & 0,38 & 0,15 \\
LHC x Ancho presa & $-0,25$ & 0,38 & & 0,26 & 0,34 \\
LCA x Volumen presa & $-0,48$ & 0,07 & & 0,57 & $0,02 *$ \\
ACA x Volumen presa & $-0,36$ & 0,20 & & 0,25 & 0,36 \\
ALTCAB x Volumen presa & $-0,34$ & 0,22 & & 0,41 & 0,12 \\
LHC x Volumen presa & $-0,31$ & 0,26 & & 0,23 & 0,38
\end{tabular}


derecho y el LHC $(n=23 ; r=0,91 ; p=0,0001)$. Solo una hembra presentó tres embriones, de acuerdo a la clasificación de LEYTON et al. (1980), corresponde a un estadio 34 de desarrollo avanzado.

La similitud en el largo hocico-cloaca $(\mathrm{mm})$ y peso (gr) entre embriones (LHC, $\mathrm{n}=3$; media $=25,3 \mathrm{~mm}$; peso media $=0,64 \mathrm{gr})$ e infantil $(\mathrm{LHC}=28 \mathrm{~mm} ; \mathrm{n}=1$ peso $=0,7$ gr) indica que en el período de fines de diciembre y principio de enero se producirían los nacimientos con un tamaño de camada de tres crías, como así también se confirma la condición vivípara en $L$. vallecurensis.

\section{DISCUSIÓN}

El nicho trófico es una de las principales dimensiones ecológicas de cualquier especie animal (PIANKA, 1973). Entre los artrópodos, $L$.vallecurensis consume principalmente ítems del orden Hymenoptera (no Formicidae). La inexistencia de diferencias en la riqueza de elementos tróficos y valores bajos de similitud en composición (Jaccard) observados entre machos, hembras y juveniles indica un alto grado de disimilitud trófica en el grupo etario y entre sexos. En machos existieron un alto grado de consumo en semillas, viéndose reflejado en un mayor valor en el índice de Simpson (dominancia).

El bajo consumo de materia vegetal por juveniles se encuentra relacionado a necesidades de crecimiento. JAKSIC \& FUENTES (1980) proponen que los juveniles a diferencia de los adultos siempre tendrían accesos a presas abundantes y económicas en relación a su tamaño. Existe un determinado tamaño el cual un lagarto puede mantener su dieta carnívora, pasando de ese tamaño, la posibilidad de capturar un insecto disminuye (Pough, 1973; JAKSIC, 1978). Este patrón se ha observado en $L$. vallecurensis, donde las hembras adultas son omnívoros y los machos con una tendencia a ser herbívoros (porcentajes altos de materia vegetal). Es probable que a los adultos les resulten más convenientes alimentarse de componentes energéticamente pobres pero abundantes como tejidos vegetales, antes que esperar la captura de presas ricas en energía (JAKSIC \& FUENTES, 1980). Antecedentes de este patrón es mencionado en Liolaemus nitidus Wiegmann, 1834 (JAKSIC \& FUENTES, 1980) y Liolaemus lutzae Mertens, 1938 (RochA, 1998).

Los machos de L. vallecurensis mostraron una dieta especialista (especies con un margen de consumo trófico estrecho), característico por el consumo de presas localmente numerosas y pequeñas. En herpetología el tipo de dieta (especialista/ oportunista) se encuentra relacionado con el modo de búsqueda de alimento. Donde los hábitos especialistas han sido relacionados con un modo de búsqueda activo y los generalistas (oportunistas) asociados con un modo de búsqueda al acecho (Huey \& PianKa, 1981). Por lo tanto en machos de L. vallecurensis la presencia de una alta numerosidad en semillas es incompatible con una forma de caza al acecho y más compatible y consistente con un modo de forrajeo activo.
En hembras y juveniles no hay una alta predominancia en numerosidad de algún ítem trófico. En ellos se encontraron un consumo menor de presas solitarias de mayor tamaño (Coleoptera) que se asocia con una especialización oportunista (valor alto del índice de Levins). Por ende las hembras y juveniles con una dieta más oportunista se consideran que pueden ser forrajeadores al acecho.

Los individuos con dietas especialistas son de gran interés en conservación. La desventaja de los organismos especialistas es que presentan estrechos límites de tolerancia, siendo estos más vulnerables que los generalistas. Además se consideran que son raros, mientras que los generalistas son más abundantes (PiANKA, 1982).

Los antecedentes mencionan que en Liolaemus wiegmannii Duméril \& Bibron, 1837 y Liolaemus saxatilis Avila \& Cei, 1992 se encuentran entre un modo de búsqueda activa y caza al acecho (AUn et al., 1999; MARTORI et al., 2002) también llamado modo mixto. La lagartija Liolaemus ruibali Donoso-Barros, 1961 con un tipo de caza al acecho (Villavicencio et al., 2005). La teoría de forrajeo se basa en la idea de que el comportamiento de alimentación es plástico y responde a diferencias en la abundancias y comportamientos de las presas (VitT \& CALDwell, 2009). Actualmente la estrategia de búsqueda de alimento constituye un continuo cuyos dos extremos están representados por estos modos (RocA, 1999). Las especies de Liolaemus se localizan en ambos extremos siendo algunos activos, otros con caza al acecho y otros mixtos.

Es probable que los machos de L. vallecurensis se comporten como especies con movimientos más exploratorios (compatible con un modo activo) que en hembras. Estas predicciones podrían ser correctas debido a que se encontró dimorfismo en la longitud del fémur siendo en machos más grande que en hembras. Esta característica se encuentra asociada con una mayor movilidad (HALLOY \& RoBles, 2002). Los machos presentan cabezas de mayores tamaños que en hembras, siendo esta característica compartida con una gran cantidad de especies de lagartos: Liolaemus olongasta Etheridge, 1993 (CÁNOVAs et al., 2006); Liolaemus cuyanus Cei \& Scolaro, 1980 (LASPIUR \& ACOSTA, 2007); Liolaemus eleodori Cei, Etheridge \& Videla, 1985 (Astudillo et al., 2015) entre otros. Una de las hipótesis planteadas en el dimorfismo sexual para las diferencias en el tamaño de cabeza menciona que debe ir acompañado de la divergencia en el comportamiento de alimentación (VINCENT \& HERREL, 2007). En L. vallecurensis solo en hembras se encontraron correlaciones significativas y positivas en el largo de cabeza con el largo, ancho y volúmenes de presas. Esto es consistente con resultados en L. eleodori, donde en hembras presentaron correlaciones con las presas consumidas (AstUDILLO et al., 2015).

La mayor distancia de separación entre los miembros encontradas en hembras posiblemente se relacionan con el tamaño abdominal asociado a la capacidad de alojar huevos (VeGA, 1999). Esta diferencia morfométrica se encuentra entre las variables más importantes y generalizadas en el dimorfismo sexual dentro del grupo Liolaemus. 
Al comparar los modos de búsquedas de alimentos (Activo/ caza al acecho) con los tamaños de cabezas se encontraron un patrón predicho por MCBRAYER \& CORBIN (2007). En L. vallecurensis el modo de búsqueda activo se asocia con un tamaño de cabeza más larga característica presente en machos. La forma de cabeza más corta en hembras está asociada con una forma de búsqueda al acecho. Sin embargo esta hipótesis también predice que un ancho estrecho está asociado a la búsqueda activa, al igual que un ancho más grande está asociado con una búsqueda al acecho (MCBRAYER \& Corbin, 2007). Este patrón con respecto al ancho de cabeza no se observó en $L$. vallecurensis.

Los datos brindados en el presente estudio en la biología reproductiva son los primeros datos. Se confirma la condición vivípara de $L$. vallecurensis y los nacimientos entre fines de diciembre y principios de enero. El volumen testicular promedio de L. vallecurensis es similar al reportado para L. elongatus en la misma época (enero) (RAmírez-PINILLA, 1992). El valor promedio testicular de L. vallecurensis para enero es mucho menor que el reportado para L. ruibali (volumen testicular $=34.5 \mathrm{~mm}^{3}$ ) (RAMÍREZ-PINILLA, 1992).

El tamaño de camada en L. vallecurensis (3 crías) es similar a otras especies; Liolaemus gracielae Abdala, Acosta, Cabrera, Villavicencio \& Marinero, 2009 (2 a 3 crías) (Acosta et al., 2008) y L. eleodori (2 a 3 crías) (CABRERA \& Monguillot, 2007). Como así también son menores a Liolaemus gravenhorsti Gray, 1845 (3 a 10) (LEYTON et al., 1980), L. ruibali (2 a 5) (RAMíREZ-PINILla, 1992), L. pictus Duméril \& Bibron, 1837 (4 a 7 crías) (IBARGÜENGOYTIA \& CUSSAC, 1996), L. elongatus (3 a 7 crías) (IBARGÜENGOYTIA \& Cussac, 1998) y Liolaemus kingii Bell, 1843 (2 a 5 crías) (IBARgüEngoytia et al., 2002). Proponemos realizar estudios temporales para conocer el ciclo reproductivo completo de esta especie.

Finalmente concluimos que no hay diferencia trófica en el principal ítems trófico consumido entre machos, hembras y juveniles. Sin embargo existe una tendencia en L. vallecurensis en usar tanto el modo activo como al acecho para la obtención del alimento, denominándose un modo de búsqueda mixto. Contrariamente a lo supuesto de que el clado Iguania son predadores al acecho (VITT \& PIANKA, 2005), en L. vallecurensis se podría considerar una especie plástica con tendencia en diferentes modos de búsqueda de alimento. Los diferentes modos para la obtención del alimento podrían ser usados para evitar la competencia intraespecífica (VitT \& CALDWELL, 2009). En el estado actual de nuestros resultados no nos es posible asignarle de manera definitiva una explicación causal a las diferencias morfológicas encontradas entre machos y hembras en L. vallecurensis. El dimorfismo sexual es el resultado de presiones selectivas (selección natural, sexual y de fecundidad), desencadenando el mismo resultado. Esto impone la necesidad de contrastar estas hipótesis como potenciales explicaciones del mismo fenómeno. Tomar a priori cualquiera de ellas como una única hipótesis aumenta el riesgo de elaborar explicaciones inapropiadas (PINCHEIRA-DonOso, 2012). Recalcamos la importancia debido a que los datos aportados en el presente trabajo sobre ecología trófica, dimorfismo sexual y biología reproductiva son los únicos con los que se cuenta actualmente en L. vallecurensis debido al difícil acceso a la localidad tipo. Destacamos la importancia por ser una especie endémica de la provincia de San Juan categorizada como vulnerable.

Agradecimientos. Agradecemos a la Subsecretaria de ambiente y desarrollo sustentable de la provincia de San Juan por los permisos autorizados para realizar la recolecta de muestras.

\section{REFERENCIAS}

Abdala, C. S.; Acosta, J. C.; Acosta, J. L.; Álvarez, B. B.; Arias, F.; Ávila, L. J.; Blanco, M. G.; Bonino, M.; Boretto, J. M.; Brancatelli, G.; Breitman, M. F.; Cabrera, M. R.; Cairo, S.; Corbalán, V.; Hernando, A.; Ibargüengoytía, N. R.; Kacoliris, F.; Laspiur, A.; Montero, R.; Morando, M.; Pelegrin, N.; Pérez, C. H. F.; Quinteros, A. S.; Semhan, R. V.; Tedesco, M. E.; Vega, L. \& Zalba, S. M. 2012. Categorización del estado de conservación de las lagartijas y anfisbenas de la República Argentina. Cuadernos de Herpetología 26:215-248.

Acosta, J. C.; Villavicencio, H. J. \& Marinero, J. A. 2008. Anfibios y Reptiles. Biodiversidad, bio-ecología y especies de valor especial para monitoreo In: Martinez-Carretero, E. ed. Diversidad Biológica y Cultural de Los Altos Andes Centrales de Argentina: línea de base de la Reserva de la Biosfera San Guillermo. San Juan, Universidad Nacional de San Juan, p. 167-179.

Anderson, R. A. \& VitT, L. J. 1990. Sexual selection versus alternative causes of sexual dimorphism in teiid lizards. Oecologia 84(2):145-157.

Astudillo, G. V.; Acosta, J. C.; Villavicencio, H. J. \& Córdoba, M. A. 2015. Ecología trófica y dimorfismo sexual del lagarto endémico Liolaemus eleodori (Iguania: Liolaemidae) del Parque Nacional San Guillermo, San Juan. Cuadernos de Herpetología 29(1):27-39.

Aun, L. \& MARTORI, R. 1998. Reproducción y dieta de Liolaemus koslowskyi Etheridge 1993. Cuadernos de Herpetología 12(1):1-9.

Aun, L.; MArtori, R. \& Rocha, C. 1999. Variación estacional de la dieta de Liolaemus wiegmanii (Squamata: tropiduridae) en un agroecosistema del sur de Córdoba, Argentina. Cuadernos de Herpetología 13(1-2):69-80.

CABrera, A. 1994. Regiones fitogeográficas Argentinas. Enciclopedia Argentina de agricultura y Jardinería, tomo II. Buenos Aires, Editorial Acme. 85p.

Cabrera, R. A. \& Monguillot, J. 2007. Liolaemus eleodori (San Guillermo's lizard): Reproduction. Herpetological Bulletin 101:34-35.

Cánovas, M. G.; Villavicencio H. J.; Acosta J. C. \& Marinero J. A. 2006. Dimorfismo Sexual y Morfometría de una Población de Liolaemus olongasta (Iguania: Liolaeminae) en la Laja, Albardón, San Juan, República Argentina. Cuadernos de Herpetología 19:57-61.

Castillo, G. N.; Villavicencio, H. J.; Acosta, J. C. \& Marinero, J. 2015. Field body temperature and temporal activity of lizards Liolaemus vallecurensis and Liolaemus ruibali in rigorous climate of the central Andes of Argentina. Multequina 24:19-31.

Castro, S. A.; Laspiur, A. \& Acosta, J. C. 2013. Variación anual e intrapoblacional de la dieta de Phymaturus cf. Palluma (Iguania: Liolaemidae) de los Andes centrales en Argentina. Revista Mexicana de Biodiversidad 84:1258-1265.

Córdoba, M. A.; Acosta, J. C.; Villavicencio, H. J. \& Astudillo, V. 2015. Análisis trófico de Phymaturus punae (Iguania: Liolaemidae): variación estacional y sexual en la región más austral de la puna Argentina. Revista Mexicana de Biodiversidad 86:1004-1013.

Cox, R. M.; Butler, M. A. \& John-Alder, H. B. 2007. The evolution of sexual size dimorphism in reptiles. In: FAIRBAIRn D. J.; BLANCKENHORN W. U. \& Székely T. ed. Sex, Size, and Gender Roles Evolutionary Studies of Sexual Size Dimorphism. Oxford, Oxford University Press, p. 38-49.

DUNHAM, A. E. 1983. Realized niche overlap, resource abundance and intensity of interspecific competition. Lizard Ecology. Cambridge, Harvard University Press. 19p.

Espinoza, R. E.; Wiens, J. J. \& TRACY, R. C. 2004. Recurrent evolution of herbivory in small, cold- climate lizards: Breaking the ecophisiological 
rules of reptilian herbivory. Proceedings of the National Academy of Sciences 101:16819-16824

FaIRBAIRN, D. J. 1997. Allometry for sexual size dimorphism: Pattern and Process in the Coevolution of Body Size in Males and Females. Annual Review of Ecology and Systematics 28:659-687.

Goldberg, S. R.; Bursey, C. R. \& Morando, M. 2004. Metazoan Endoparasites of 12 Species of Lizards from Argentina. Comparative Parasitology 71(2):208-214.

GonzÁlez, C. G.; Felpeto, A. B.; Estraviz, I. M.; Alarcón, I. R.; CastaÑo, A. R. V. \& Liste, A. V. 2006. Tratamientos de datos. Madrid, Edición Díaz de Santos. 356p.

Halloy, M. \& Robles, C. 2002. Spatial distribution in a Neotropical lizard, Liolaemus quilmes (Liolaemidae): site fidelity and overlapping among males and females. Bulletin of the Maryland Herpetological Society 38:118-129.

Hedrick, A. V. \& Temeles, E. J. 1989. The evolution of sexual dimorphism in animals: hypotheses and tests. Trends in Ecology \& Evolution 4(5):136-138.

Huey, R. B. \& Pianka, E. R. 1981. Ecological consequences of foraging mode. Ecology 62(4):991-999.

IBARgÜEngoytía, N. R. \& CusSAC, V. E. 1996. Reproductive biology of the viviparous lizard, Liolaemus pictus (Tropiduridae): biennial female reproductive cycle? Herpetological Journal 6:137-144.

Ibargü̈Engoytia, N. R. \& CussaC, V. E. 1998. Reproduction of the viviparous lizard Liolaemus elongatus in the highlands of southern South America: plastic cycles in response to climate? Herpetological Journal 8:99-106.

Ibargüengoytía, N. R.; Halloy, M. \& Crocco, M. C. 2002. El parto en el lagarto Liolaemus kingii (Iguania: Liolaemidae): observaciones etológicas. Cuadernos de Herpetología 16(2):129-135.

JAKSIC, F. M. 1978. ¿A qué tamaño se hace herbívora una lagartija. Anales del Museo de Historia Natural de Valparaíso 11:113-115.

JAKSIC, F. M. \& Fuentes E. R. 1980. Observaciones autoecológicas en Liolaemus nitidus (Lacertilia: Iguanidae). Studies on Neotropical Fauna and Environment 15:109-124.

LaSPIUR, A. \& AcostA, J. C. 2007. Dimorfismo Sexual de Liolaemus cuyanus Cei and Scolaro, 1980 (Iguania: Liolaemidae) en una población de San Juan, Argentina. Revista Peruana de Biología 14:47-50.

Leyton, V.; Miranda, E. \& Bustos-OBregon, E. 1980. Gestational Chronology in the viviparous lizard Liolaemus gravenhorsti (Gray) with remarks on ovarian and reproductive activity. Archives of Biology 91:347-361.

Martori, R.; JuÁrez, R. \& Aun, L. 2002. La taxocenosis de lagartos de Achiras, Córdoba, Argentina: parámetros biológicos y estado de conservación. Revista Española de Herpetología 16:73-91.

McBrayer, L. D. \& Corbin, C. E. 2007. Patterns of head shape variation in lizard: morphological correlates of foraging mode. In: REILLY, S. M.;
McBrayer, L. D. \& Miles, D. B. ed. Lizard ecology. The Evolutionary Consequences of Foraging Mode. New York, Cambridge University Press, p. 271-301.

MoRENO, C. E. 2001. Manual de métodos para medir la biodiversidad. Xalapa, Universidad Veracruzana. 83p.

Pereyra, E. A. 1992. Nueva especie de lagarto andino: Liolaemus vallecurensis (Tropiduridae, Liolaeminae). Noticiario Mensual del Museo Nacional de Historia Natural 322:10-14.

Perucca, L. P. \& Martos, L. M. 2009. Análisis preliminar de la evolución del paisaje cuaternario en el valle de Iglesia, San Juan. Revista de la Asociación Geológica Argentina 65(4):624- 637.

Pianka, E. R. 1982. Ecología evolutiva. Barcelona, Omega. 365p.

PianKa, E. R. 1973. The structure of lizard communities. Annual Review of Ecology and Systematics 4:53-74.

Pincheira-Donoso, D. 2012. Selección y evolución adaptativa. Fundamentos teóricos y empíricos desde la perspectiva de los lagartos. Santiago, Ediciones Universidad Católica de Chile. 403p.

Pinkas, L.; Oliphanat, M. \& Iverson, Z. 1971. Food habits of albacore bluefin tuna and bonito in California waters. Fish Bulletin 152:1-105.

Pough, F. A. 1973. The lizard energetics and diet. Ecology 54:837-844.

Ramírez-Pinilla, M. P. 1992. Actividad reproductiva en tres especies simpátricas del género Liolaemus (Reptilia: Sauria: Tropiduridae). Caldasia 17(1):67-74.

Roca V. 1999. Relación entre las faunas endoparásitas de reptiles y su tipo de alimentación. Revista Española de Herpetología 13:101-121.

Rocha, C. F. D. 1998. Ontogenetic shift in the rate of plant consumption in a tropical lizard (Liolaemus lutzae). Journal of Herpetology 32(2):274-279.

SOKaL, R. R. \& RoHLF, F. J. 1999. Introducción a la bioestadística. Barcelona, Editorial Reverté, S.A. 380p.

TELLERÍA, J. L. 1986. Manual para el censo de los vertebrados terrestres. Madrid, Editorial Raíces. 32p.

Vega, L. E. 1999. Ecología trófica de Liolaemus multimaculatus (Sauria: Tropiduridae). Bolletino del Museo Regionale di Scienze Naturali di Torino 16:27-38.

Villavicencio, H. J.; Acosta, J. C. \& Cánovas, M. G. 2005. Dieta de Liolaemus ruibali Iguanidae: Liolaeminae) en la reserva de usos múltiples Don Carmelo, San Juan, Argentina. Multequina 14:47-52.

Vincent, S. E. \& Herrel, A. 2007. Functional and ecological correlates of ecologically-based dimorphisms in squamate reptiles. Integrative and Comparative Biology 47(2):172-188.

VitT L. J. \& CALDWELL J. P. 2009. Herpetology, An Introductory Biology of Amphibians and Reptiles. 3ed. London, Elsevier. 697p.

VitT L. J. \& PiANKA E. R. 2005. Deep history impacts present-day ecology and biodiversity. Proceedings of National Academy of Science 102:7877-7881.

ZAR, J. 1996. Biostatistical analysis. 3ed. New Jersey, Prentice-Hall. 663p. 